\title{
SCIENTIFIC REPORTS

\section{Induced DNA hypomethylation by Folic Acid Deprivation in Bovine Fibroblast Donor Cells Improves Reprogramming of Somatic Cell Nuclear Transfer Embryos}

\author{
Mina Jozi ${ }^{1}{ }^{1}{ }^{2}$, Farnoosh Jafarpour ${ }^{1 *}$, Reza Moradi ${ }^{1}$, Faezeh Ghazvini Zadegan ${ }^{1}$, \\ Khadijeh Karbalaie $^{3}$ \& Mohammad Hossein Nasr-Esfahani ${ }^{1 *}$
}

Aberrant patterns of DNA methylation are consistent events in SCNT derived embryos and mechanistically are believed to be related to abnormal development. While some epigenetic drugs have been used in attempts to improve SCNT efficiency but some concerns remained toward the safety of these drugs on the health of future offspring. Folate is an essential cofactor in one-carbon cycle for conversion of homocysteine to methionine, thereby ensuring supply of SAM, the universal methyl donor for many biological methylation reactions including DNA methylation. Therefore, in vitro DNA hypo-methylation can be induced by folate deprivation and this study aims at deciphering the role of folic acid deprivation in culture medium of BFFs for 6 days on SCNT efficiency. Our data revealed that culture of fibroblast cells in folate-medium containing $0.5 \%$ FBS did not alter the cell cycle compared to other groups. Flowcytometric analysis revealed that DNA methylation (5-mC level) in folate deprived cells cultured in $0.5 \%$ serum was decreased compared to folate+ group. The result of bisulfite sequencing was in accordance with flowcytometric analysis, which indicated a decrease in DNA methylation of POU5F1 promoter. Gene expression analysis revealed an increase in expression of POU 5F1 gene in folate - group. The nuclear area of the cells in folate-group was significantly larger than folate + group. Induced DNA hypomethylation by folate deprivation in the folate - group significantly improved blastocyst rate compared to the folate+ group. DNA methylation level in POU5F1 promoter and ICR of $H 19$ and IGF2 of SCNT derived embryos in the folate-group was similar to the IVF derived blastocysts. In conclusion, our results proposes a promising "non-chemical" instead of "chemical" approach using inhibitors of epigenetic modifier enzymes for improving mammalian SCNT efficiency for agricultural and biomedical purposes.

Somatic cell nuclear transfer (SCNT) technique, as an alternative counterpart for fertilization, comprises erasing epigenetic memory of somatic cell nucleus and establishing new epigenetic information and conferring a totipotent state to the newly forming embryos ${ }^{1}$. The birth of the well-known "Dolly the sheep", as the result of the first successful SCNT in mammalian species was a metaphor for demonstration of developmental plasticity ${ }^{2}$.

Despite the fact that several mammalian species have been cloned through SCNT technique, the overall efficiency of this technique has remained remarkably low, approximately less than $5 \%$ in many species ${ }^{3}$. Generally, SCNT derived embryos suffer from abnormal epigenetic reprogramming, such as DNA/histone hyper-methylation and histone hypo-acetylation. These anomalies hamper early and late embryonic development $^{4,5}$. Based on background literature the most important cause of abnormal epigenetic reprogramming in

\footnotetext{
${ }^{1}$ Department of Reproductive Biotechnology, Reproductive Biomedicine Research Center, Royan Institute for Biotechnology, ACECR, Isfahan, Iran. ${ }^{2}$ ACECR Institute of Higher Education (Isfahan Branch), Isfahan, Iran. ${ }^{3}$ Department of Cellular Biotechnology at Cell Science Research Center, Royan Institute for Biotechnology, ACECR, Isfahan, Iran. *email: jafarpour.farnoosh@gmail.com; mh.nasr-esfahani@royaninstitute.org
} 


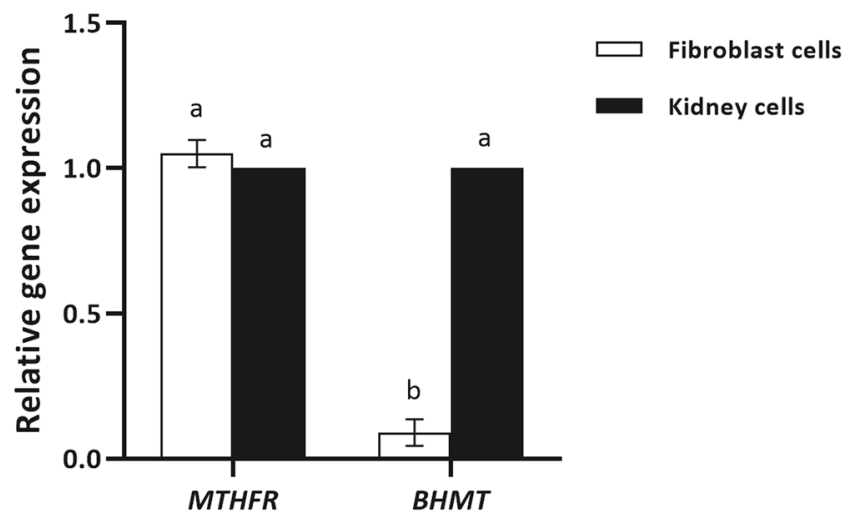

Figure 1. Real-time reverse-transcriptase PCR gene expression analysis of $M T H F R$ and BHMT in fibroblast cells derived from skin and kidney in bovine. Fold-change values were calculated from triplicate technical replicates of three biological replicates following normalization to $\beta$-ACT. Values are means \pm SEM. Different letters indicate significant differences $(P<0.05)$.

SCNT embryos is the specific epigenetic characteristic of somatic donor cells, which is very far from the specialized epigenetic status of sperm and oocyte.

One of the most well-known approaches for improving epigenetic reprogramming of reconstructed embryos during SCNT is removal of epigenetic barriers in genome of donor cells and/or reconstructed embryos using epigenetic chemical modifiers or drugs. In addition to this approach, siRNAs targeting of transcripts of epigenetic modifier enzymes remains an alternative approach. Till now, many epigenetic drugs such as DNA methyltransferase inhibitors (DNMTis) and histone deacetylase inhibitors (HDACis) have been used to improve in vitro and in vivo development of SCNT embryos ${ }^{6-9}$. These two categories of epigenetic modifiers by inducing DNA hypo-methylation and histone hyper-acetylation result in chromatin relaxation and thereby improves nuclear reprogramming.

While some of these epigenetic drugs have remarkably improved the pre- and post-implantation development of SCNT derived embryos ${ }^{6-9}$, but we have some concerns about the side effects of these drugs on the health of future offspring, which remained to be elucidated. Therefore, designing a non-chemical approach which can induce DNA hypo-methylation and/or histone hypo-methylation/hyper-acetylation in donor cells and/or reconstructed embryos is of great interest and importance.

S-adenosyl methionine (SAM) is the predominant methyl donor for many biological methylation reactions including DNA methylation and histone methylation in mammalian cells ${ }^{10}$. In one carbon cycle, remethylation of homocysteine can be carried out via two pathways. In the most common pathway, operating in somatic cells, a methyl group derived from serine, carried by methyl tetrahydrofolate, is transferred to homocysteine by methylenetetrahydrofolate reductase enzyme (MTHFR). In an alternative pathway of methionine production restricted to liver and kidney cells in humans, a methyl group is transferred directly from betaine to homocysteine by betaine-homocysteine methyltransferase (BHMT) enzyme ${ }^{11,12}$. Subsequently, methionine is converted to SAM by addition of adenosine triphosphate by methionine adenosyltransferase ${ }^{13}$.

Researchers have shown that any mutation in MTHFR gene or in vivo deficiency of folate leads to DNA hypo-methylation in genomic DNA, which may predispose the individuals to various cancers ${ }^{14}$. Furthermore, in vitro folate deprivation result in a significant genomic DNA hypo-methylation in non-transformed cell lines ${ }^{15}$.

Considering that in vitro folate deprivation, can induce DNA hypo-methylation this study aims at deciphering the role of folic acid deprivation in culture medium of bovine fibroblast donor cells (BFFs) for 6 days on SCNT efficiency.

\section{Results}

Bovine fetal fibroblast cells only exhibit expression of MTHFR enzyme. Since in this study we aimed to determine the effect of induced DNA hypo-methylation in fibroblast cells on SCNT efficiency by folate deprivation, mRNA expression of MTHFR and BHMT mRNA were assessed in both fibroblast and kidney cells to confirm that the only active pathway for methionine production in fibroblast cells is MTHFR. In mammalian kidney and liver, homocysteine can also be converted to methionine by two distinct pathways using MTHFR and BHMT enzymes while in other tissues only MTHFR enzyme is responsible for production of methionine.

Analysis of relative mRNA expression levels of MTHFR and BHMT in bovine fibroblast and kidney cells by independent samples t-test revealed a significant lower level of BHMT mRNA expression in fibroblast cells relative to MTHFR $(P<0.05$, Fig. 1). Furthermore, gene expression analysis revealed a significant higher expression of $B H M T$ in kidney cells versus fibroblast cells $(P<0.05$, Fig. 1$)$. These data demonstrated that mRNA transcripts for BHMT enzyme is likely to be absent in fibroblast somatic cells in compare to kidney cells and the likely active pathway for methionine production in fibroblast cells appear to be MTHFR.

Folate deprivation does not reduce fibroblast proliferation. Previously, several studies have shown that folate deprivation reduces proliferation of various cell types. To determine whether folate deprivation can affect the proliferation of fibroblast cells, the MTS assay was carried out in presence of $10 \%$ and $0.5 \%$ FBS. Our 


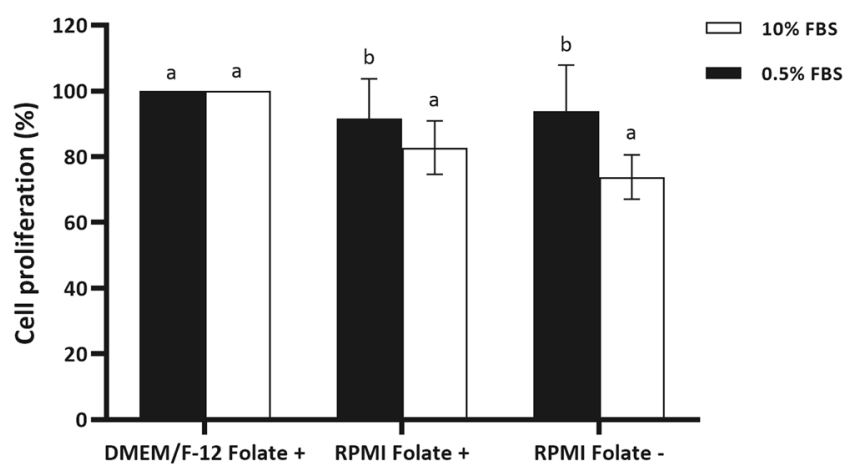

Figure 2. Cell proliferation of BFF cells cultured in DMEM/F-12 Folate + , RPMI Folate + and RPMI Folate - in presence of 10 (white bar) and $0.5 \%$ (black bar) FBS for 6 days. The results were normalized to DMEM/F-12 Folate + group and are presented as the mean \pm SEM. Different letters indicate significant differences $(P<0.05)$.
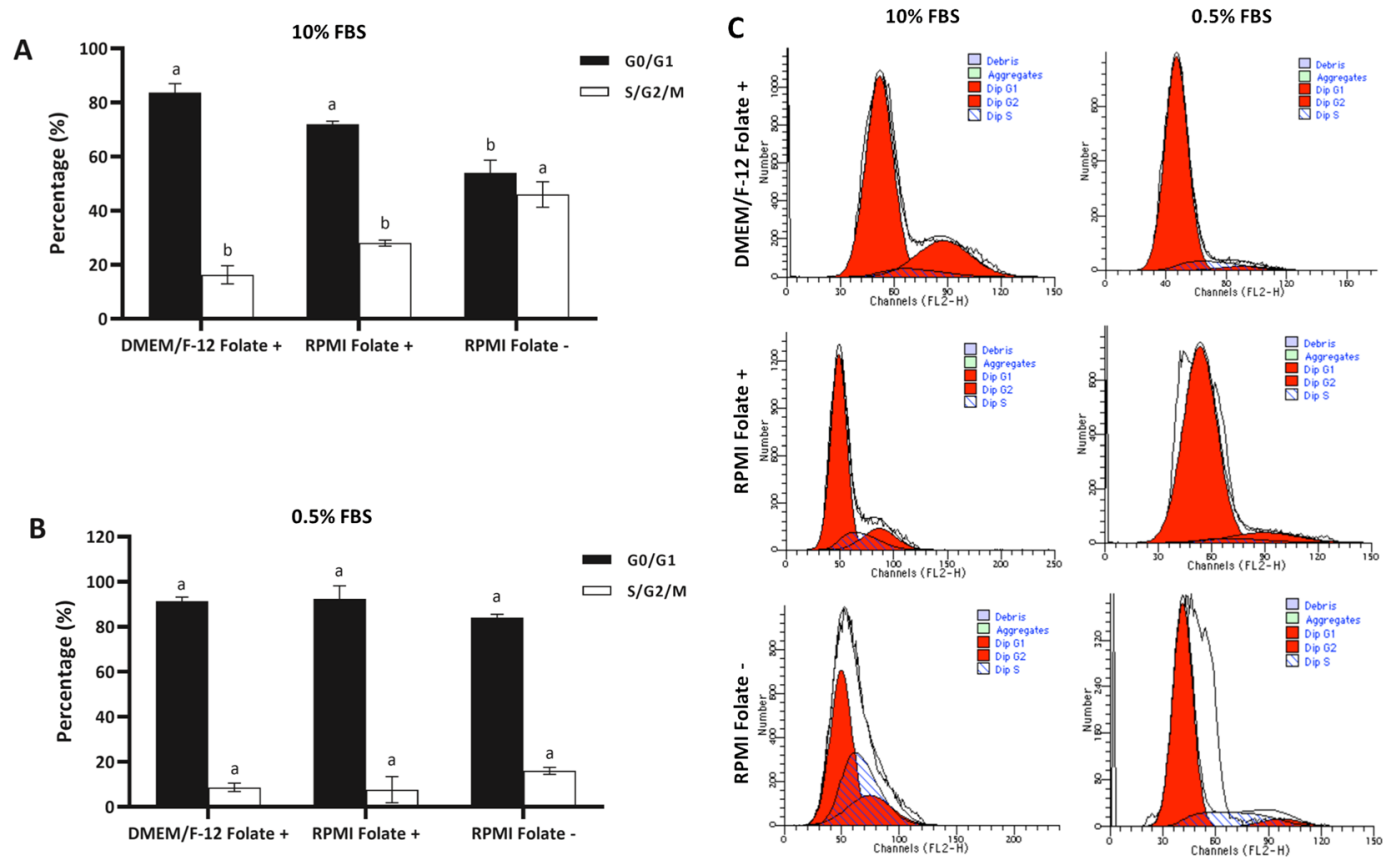

Figure 3. Cell cycle distribution of BFF cells propagated in DMEM/F-12 Folate+, RPMI Folate+ and RPMI Folate - in presence of (A) 10 and (B) 0.5\% FBS growth medium for 6 days. (C) Flow cytometry histograms indicating the cell cycle distribution of above mentioned BFF cells. Different letters indicate significant differences $(\mathrm{P}<0.05)$.

results revealed that about 6 days of culture in RPMI 1640 medium, both folate sufficient and folate deficient, decreased proliferation rate in compare to DMEM/F-12 folate sufficient medium, routinely used for cell proliferation and starvation in SCNT procedure, in presence of $10 \%$ serum $(P<0.05$, Fig. 2$)$. Furthermore, RPMI 1640 folate deficient medium did not affect the proliferation rate relative to RPMI 1640 folate sufficient medium either in presence of $10 \%$ or $0.5 \%$ serum $(P>0.05$, Fig. 2$)$. Finally, neither type of culture medium (DMEM/F-12 or RPMI 1640) nor folate level (folate sufficient medium or folate deficient medium) affected proliferation rate in presence of $0.5 \%$ FBS $(P>0.05$, Fig. 2). All the comparisons were done by one-way ANOVA analysis $(\alpha=0.05)$ followed by Tukey HSD test.

Folate deprivation induces arrest in S-phase in fibroblast cells. To determine the effect of folate deficiency on cell cycle of BFF cells, cell cycle analysis was performed after 6 days of culture. It was shown that cells lacking folate accumulate in the $S$ phase possibly due to nucleotide imbalance and slow DNA synthesis. As depicted in Fig. 3A,C, analysis of cell cycle of cells cultured in DMEM/F-12 folate sufficient medium by one-way ANOVA followed by Tukey HSD test revealed a cell cycle distribution similar to that of cells cultured in RPMI 1640 folate sufficient medium in presence of $10 \%$ FBS $(P>0.05)$, whereas cells cultured in RPMI 1640 folate deficient medium showed a significant increase in the proportion of cells in the $S$ phase in presence of $10 \%$ FBS 


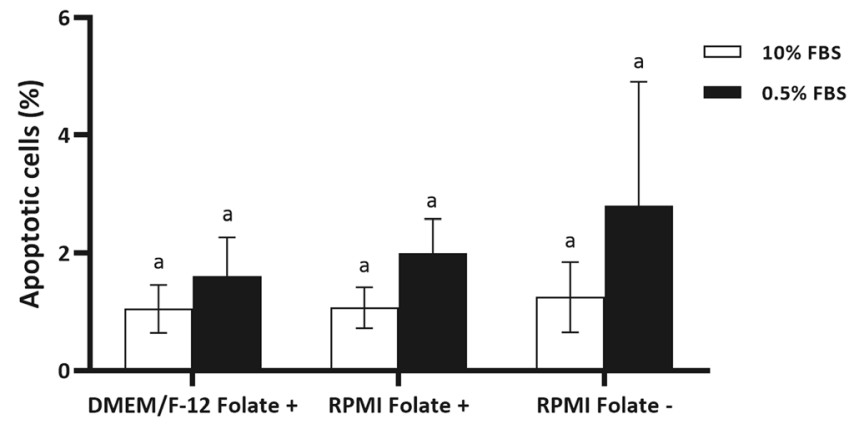

Figure 4. Analysis of apoptotic cells by TUNEL staining of BFF cells propagated in DMEM/F-12 Folate+, RPMI Folate + and RPMI Folate - in presence of 10 (white bar) and 0.5\% (black bar) FBS for 6 days. Values represent the mean \pm SEM number of TUNEL-positive cells. Different letters indicate significant differences $(\mathrm{P}<0.05)$.

A

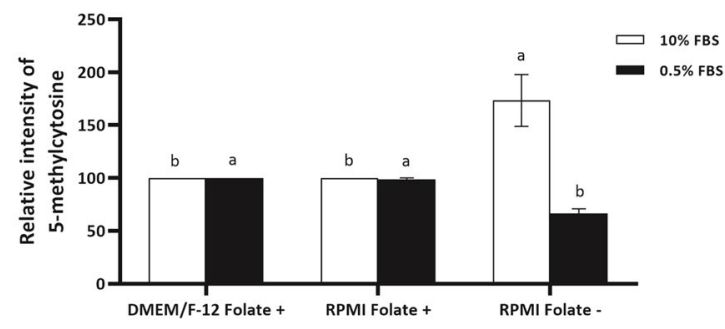

B
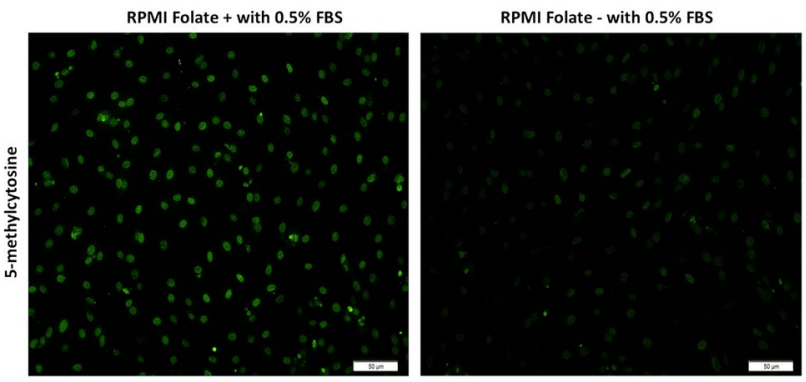

Figure 5. (A) Flowcytometry assay for relative level of DNA methylation was performed on BFF cells propagated in DMEM/F-12 Folate+, RPMI Folate+ and RPMI Folate - in presence of 10 (white bar) and $0.5 \%$ (black bar) FBS for 6 days. Values represent the mean \pm SEM. Different letters indicate significant differences $(\mathrm{P}<0.05)$. (B) Immunostaining of BFF cells propagated in RPMI Folate + and RPMI Folate - in presence of $0.5 \% \mathrm{FBS}$ for $5-\mathrm{mC}$. Scale bars represent $50 \mu \mathrm{m}$.

$(P<0.05$, Fig. 3$)$, suggesting that folate-deprived cells have an impaired capacity to synthesize DNA and to complete the cell cycle. Furthermore, in presence of $0.5 \%$ serum, presence or absence of folate in culture medium did not affect the distribution of various stages in cell cycle $(P>0.05$, Fig. 3B,C).

Folate deprivation does not increase DNA damage in fibroblast cells. Folate deprivation has been linked to increased DNA breaks owing to excessive uracil incorporation. DNA double-strand breaks is the most damaging DNA breaks in terms of genome integrity. In this study, terminal deoxynucleotidyl transferase-mediated dUTP nick-end labelling (TUNEL) immunostaining was carried out to detect cellular DNA fragmentation and to assess apoptosis in fibroblast cells cultured in folate deficient culture medium. Analysis of apoptotic cells by one-way ANOVA followed by Tukey HSD test revealed no significant elevation in TUNEL positive cells in presence or absence of folate in culture medium (DMEM/F-12 Folate + vs. RPMI Folate+ vs. RPMI Folate -$)$ in presence of $10 \%$ and $0.5 \%$ serum $(P>0.05$, Fig. 4$)$.

Effect of folate deprivation on global DNA methylation. The impact of folate deprivation on global DNA methylation in BFF cells was investigated using quantitative flow cytometry. BFF cells treated with folate sufficient and deficient culture medium in presence of $10 \%$ and $0.5 \%$ FBS for 6 days were used for detection of global DNA methylation.

The flow cytometry data analysed by one-way ANOVA followed by Tukey HSD test suggested a significantly lower degree of genomic DNA methylation in the folate deprived cells, compared with the folate-sufficient cells in presence of $0.5 \%$ serum $(P<0.05$, Fig. $5 \mathrm{~A}, \mathrm{~B})$. Interestingly, we observed higher degree of genomic DNA 
A
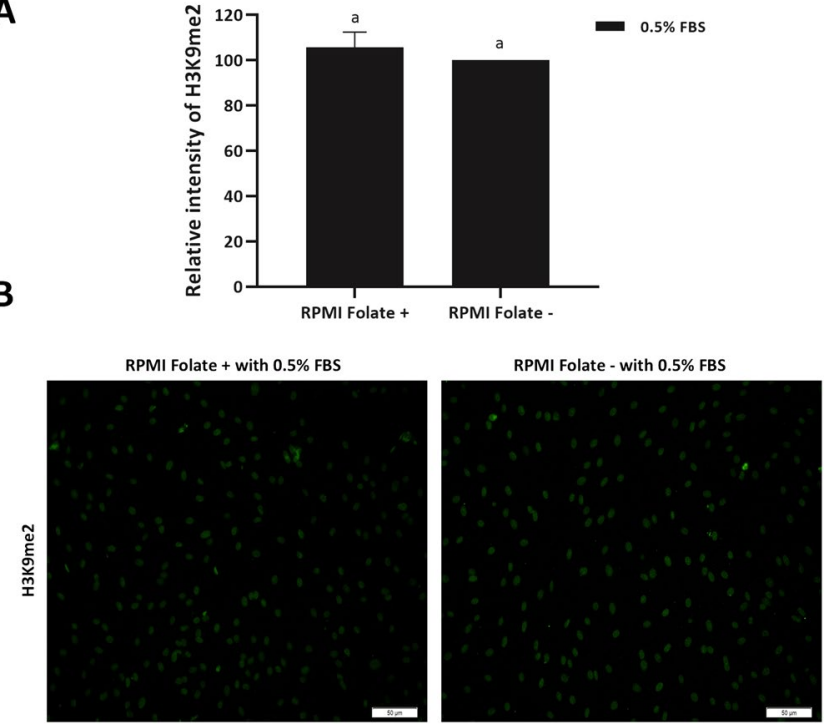

Figure 6. (A) Flowcytometry assay for relative $\mathrm{H} 3 \mathrm{~K} 9 \mathrm{me} 2$ was performed on BFF cells propagated in RPMI Folate + and RPMI Folate - in presence of $0.5 \%$ FBS for 6 days. Values represent the mean \pm SEM. Different letters indicate significant differences $(\mathrm{P}<0.05)$. (B) Immunostaining of BFF cells propagated in RPMI Folate+ and RPMI Folate- in presence of $0.5 \%$ FBS for $\mathrm{H} 3 \mathrm{~K} 9 \mathrm{me} 2$. Scale bars represent $50 \mu \mathrm{m}$.

methylation in the folate deprived cells, compared with the folate sufficient cells in presence of $10 \%$ serum $(P<0.05$, Fig. 5A). The mechanism of this phenomenon is unknown and needs to be studied in detail.

Effect of folate deprivation on $\mathrm{H} 3 \mathrm{~K} 9 \mathrm{me} 2$. The impact of folate deprivation on $\mathrm{H} 3 \mathrm{~K} 9 \mathrm{me} 2$ in $\mathrm{BFF}$ cells was investigated using quantitative flow cytometry and data analysed by independent samples t-test. Interestingly, we observed that the value for $\mathrm{H} 3 \mathrm{~K} 9 \mathrm{me} 2$ remained unchanged following 6 days folate deprivation in culture medium in presence of $0.5 \%$ FBS (RPMI Folate+ vs. RPMI Folate -$)(P>0.05$, Fig. 6A). In addition, immunocytochemistry of BFF cells revealed that intensity of $\mathrm{H} 3 \mathrm{~K} 9 \mathrm{me} 2$ in RPMI Folate+ group is similar to RPMI Folate-group (Fig. 6B).

Fibroblast cells in folate deficient medium demonstrate larger nuclear area than their counterparts in folate sufficient medium. We compared mean nuclear area in fibroblast cells serum starved in folate deficient medium against their counterparts in folate sufficient medium. By staining cells with Hoechst 33342 and measuring the nuclear area with ImageJ software and analysis by one-way ANOVA followed by Tukey HSD test, we found that mean nuclear area in fibroblast cells in folate deficient medium is significantly higher than fibroblast cells in folate sufficient medium (DMEM/F-12 and RPMI 1640 medium) $(P<0.05$, Fig. 7A,B), suggesting a more relaxed chromatin configuration in folate deficient culture medium. Furthermore, our data revealed a significant smaller mean nuclear area in RPMI 1640 folate sufficient medium against to fibroblast cells in DMEM/F-12 folate sufficient culture medium $(P<0.05$, Fig. 6), indicating a more compact chromatin state of fibroblast cells in RPMI 1640 medium in compare to DMEM/F-12 medium.

Fibroblast cells in folate deficient medium demonstrate lower genomic DNA methylation in promoter of POU5F1 gene but not in ICR of H19/IGF2 gene and promote expression of POU5F1. Following the initial characterization of global DNA methylation in fibroblast cells in folate deficient culture medium by flow cytometry, we examined DNA methylation level of ICR of H19/IGF2 (an imprinting gene) (Fig. 8A) and POU5F1 promoter (a non-imprinting gene) (Fig. 8B) using bisulfite sequencing analysis and data were analysed by independent samples t-test. In addition, mRNA expression of assessed genes was analysed by independent samples t-test. Meanwhile, culture of fibroblast cells in folate deficient medium for 6 days significantly reduced DNA methylation level of $P O U 5 F 1$ promoter $(P<0.05$, Fig. 8C), consequently leading to increased expression levels of POU5F1 $(P<0.05$, Fig. 8D). However, no differences in DNA methylation level $(P>0.05$, Fig. 8C) and gene expression of $H 19$ and $I G F 2$ imprinted genes $(P>0.05$, Fig. 8D) were observed between the fibroblast cells in folate deficient medium and their counterparts in folate sufficient medium. All together, these results suggested that folate deficient medium can initiate DNA demethylation and promote expression of $P O U 5 F 1$ in bovine fibroblast cells. In addition, no changes were observed in the expression levels of $D N M T 3 A$ and DNMT3B between the fibroblast cells cultured in folate sufficient and deficient medium $(P>0.05$, Fig. 8D).

Reconstructed oocytes by fibroblast cells cultured in folate deficient medium exhibited higher developmental potential than those cultured in folate sufficient medium. Using BFF cells treated for 6 days in folate deficient culture medium (RPMI 1640 Folate -) as somatic donor cells, we generated SCNT embryos and examined their preimplantation development in compare to those generated by fibroblast cells 
A

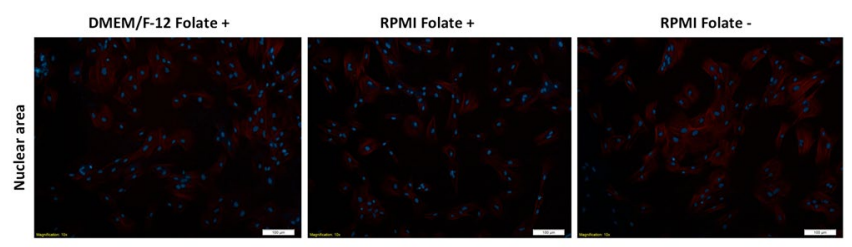

B

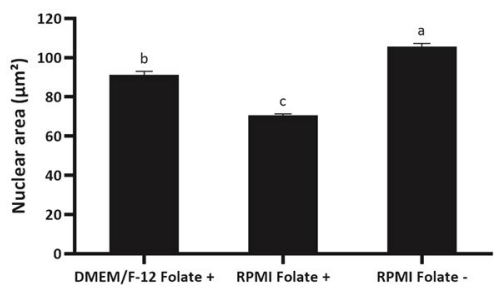

Figure 7. (A) The nuclear area of folate deprived cells was assessed by fluorescence microscopy after phalloidintetramethylrhodamine B isothiocyanate and Hoechst 33342 staining. Merged images of tubulin and DNA are shown. Scale bars represent $100 \mu \mathrm{m}$. (B) Using ImageJ software (National Institute of Mental Health, Bethesda, Maryland, USA) the nuclear area of 100 fibroblasts in 10 different fields was calculated. Values represent the mean \pm SEM. Different letters indicate significant differences $(\mathrm{P}<0.05)$.

A

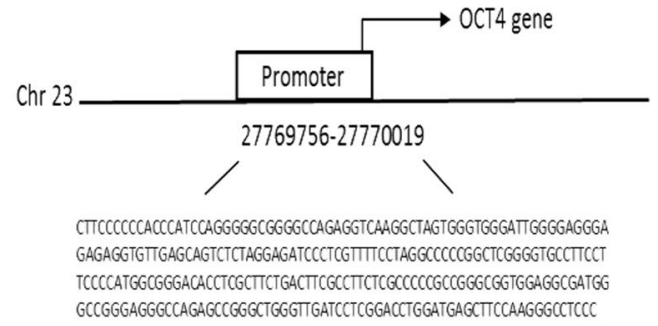

B

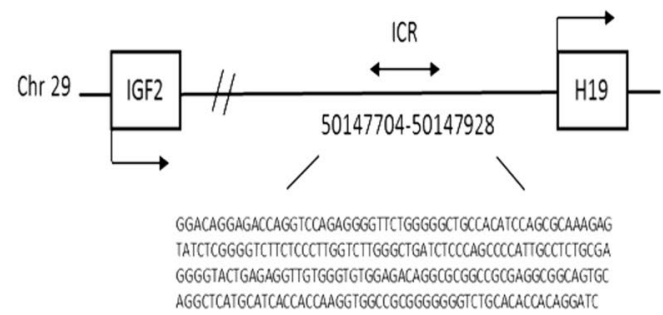

C

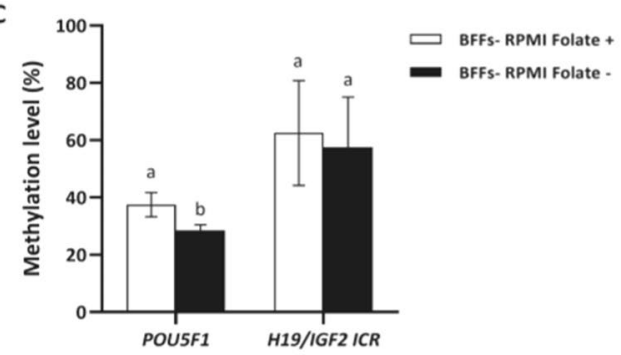

D

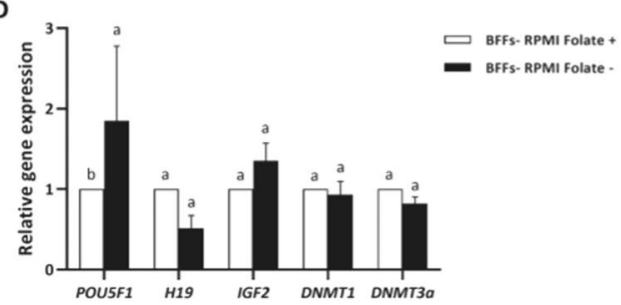

Figure 8. Schematic representation of the bovine (A) H19/IGF2 ICR region and (B) POU5F1 promoter with gene sequences used for methylation sequencing. (C) Quantitative analysis of $5 \mathrm{mC}$ levels in promoter of POU5F1 gene and ICR of H19/IGF2 imprinted genes in BFF cells propagated in RPMI Folate+ and RPMI Folate - culture medium in presence of $0.5 \%$ serum for 6 days. (D) RT-qPCR analysis of POU5F1, H19, IGF2, DNMT1 and DNMT3A expression in BFF cells propagated in RPMI Folate + and RPMI Folate - culture medium in presence of $0.5 \%$ serum for 6 days. Values represent the mean \pm SEM. Different letters indicate significant differences $(\mathrm{P}<0.05)$.

cultured for 6 days in folate sufficient medium (DMEM/F-12 Folate+ and RPMI 1640 Folate + ). Analysis of cleavage and blastocyst rates by one-way ANOVA followed by Tukey HSD test revealed that while there was no difference between the cleavage rate of DMEM/F-12 Folate+, RPMI 1640 Folate + and RPMI 1640 Folate - groups but the RPMI 1640 Folate - group demonstrated significant higher blastocyst yield (26.56 \pm 2.90$)$ RPMI 1640 Folate $+(13.83 \pm 3.64)$ group $(P<0.05$, Table 1$)$. Blastocyst yield in RPMI 1640 Folate - group, however, was not significantly higher than DMEM/F-12 Folate + group $(21.58 \pm 3.91)(P>0.05$, Table 1$)$.

Treating of fibroblast cells with folate deficient culture medium can partially rectify the hyper-methylation state of POU5F1 promoter gene and promote its expression in SCNT embryos but not in H19/IGF2 gene. We also detected DNA methylation level of POU5F1 promoter and ICR of H19/IGF2 imprinted gene in blastocyst embryos of IVF, SCNT-RPMI Folate+, and SCNT-RPMI Folategroups by bisulfite sequencing assay and data were analysed by one-way ANOVA followed by Tukey HSD test. 


\begin{tabular}{|l|l|l|l|l|}
\hline & & & Embryo development \\
\cline { 4 - 5 } Groups & \multirow{2}{*}{$\begin{array}{l}\text { No. of } \\
\text { replicates }\end{array}$} & $\begin{array}{l}\text { No. of embryos } \\
\text { cultured }\end{array}$ & $\begin{array}{l}\text { No. of Cleaved } \\
\text { embryos (\%) }\end{array}$ & $\begin{array}{l}\text { No. of blastocysts on } \\
\text { day 7 (\%) }\end{array}$ \\
\hline DMEM/F-12 Folate+ & 5 & 128 & $101(85.97 \pm 5.08)^{\mathrm{a}}$ & $18(21.58 \pm 3.91)^{\mathrm{ab}}$ \\
\hline RPMI Folate+ & 7 & 167 & $128(81.54 \pm 2.76)^{\mathrm{a}}$ & $19(13.83 \pm 3.64)^{\mathrm{b}}$ \\
\hline RPMI Folate- & 9 & 253 & $208(87.66 \pm 1.79)^{\mathrm{a}}$ & $52(26.56 \pm 2.90)^{\mathrm{a}}$ \\
\hline
\end{tabular}

Table 1. Effect of folate deprivation on development competence of the SCNT derived embryos.

A

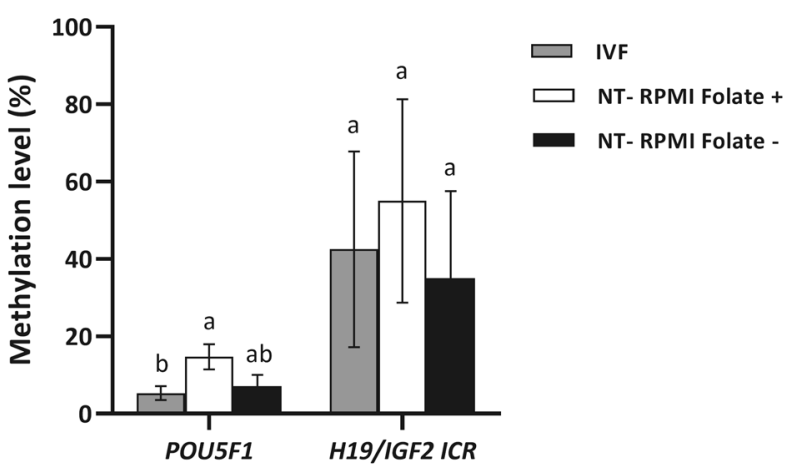

B

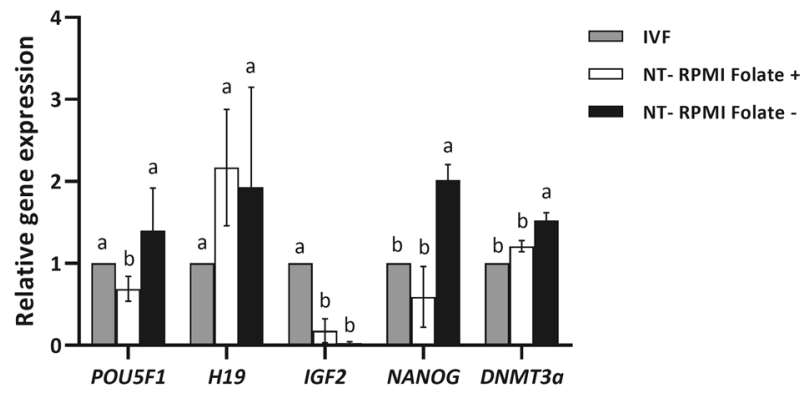

Figure 9. (A) Quantitative analysis of $5 \mathrm{mC}$ levels promoter of POU5F1 gene and ICR of H19/IGF2 imprinted genes in blastocyst embryos in IVF, NT-RPMI Folate+, and NT-RPMI Folate- groups. (B) RT-qPCR analysis of POU5F1, H19, IGF2, NANOG and DNMT3A expression in IVF, NT-RPMI Folate+, and NT-RPMI Folate at blastocyst stage. Values represent the mean \pm SEM. Different letters indicate significant differences $(\mathrm{P}<0.05)$.

The DNA methylation level of POU5F1 promoter in SCNT-RPMI Folate+ was significantly higher than IVF and SCNT-RPMI Folate - $(P<0.05$, Fig. 9A), whereas this level in SCNT-RPMI Folate - group was similar to IVF group $(P>0.05$, Fig. 9A). The DNA methylation level of H19/IGF2 ICR was similar between IVF, SCNT-RPMI Folate + and SCNT-RPMI Folate - groups $(P<0.05$, Fig. 9A $)$.

To further confirm the regulatory relationship between folate deprivation and embryonic development, we performed RT-qPCR to detect expressions of key pluripotency genes (POU5F1 and NANOG) and imprinted genes (H19 and IGF2) in blastocysts of IVF, SCNT-RPMI Folate+, and SCNT-RPMI Folate - groups. Analysis of the assessed genes by one- way ANOVA followed by Tukey HSD test revealed significantly lower level of expression of POU5F1 and NANOG in the SCNT- RPMI Folate+ group relative to that of IVF blastocysts and SCNT-RPMI Folate - group $(P<0.05$, Fig. 9B). However, SCNT- RPMI Folate - blastocysts showed POU5F1 and NANOG mRNA level approximating that of the IVF group $(P>0.05$, Fig. 9B). We also noted that H19 mRNA level did not show significant difference among the three aforementioned groups $(P>0.05$, Fig. 9B).

These findings suggested that treatment of donor fibroblast cells with folate deficient culture medium can promote POU5F1 and NANOG expression in SCNT embryos.

\section{Discussion}

In this study, we developed a non-chemical approach by altering DNA methylation of fibroblast donor cells by deprivation of folic acid in cell culture medium, and we acknowledged that deprivation of folic acid for 6 days in presence of $0.5 \%$ FBS improves developmental competence of bovine SCNT embryos.

In order to make sure that the only mechanism for producing methionine in fibroblast cells is MTHFR enzyme, and folic acid deprivation can efficiently induce DNA hypo-methylation in fibroblast cells, we assessed the expression of MTHFR and BHMT enzymes in fibroblast cells. In consistent with previous studies ${ }^{11,16}$, our data revealed that BHMT enzyme is likely to be absent or very low in fibroblast somatic cells and the only active pathway for methionine production in fibroblast cells appear to be MTHFR. 
In examining the effect of folic acid deprivation on SCNT efficiency, the type of culture medium used is very important. DMEM/F-12 is a common culture medium for culturing fibroblast cells but unfortunately, DMEM/F-12 folic acid free medium is not available. Therefore, we focused on RPMI 1640 medium with and without folic acid for assessing the effect of folic acid deprivation on SCNT efficiency. Considering that DMEM/F-12 is a common culture medium for culturing fibroblast cells, thereby, we used all three culture medium (DMEM/F-12 Folate+, RPMI 1640 Folate+ and RPMI 1640 Folate-) throughout our study.

The effect of folic acid free culture medium (RPMI 1640 Folate-) for 6 days in presence of $10 \%$ serum surprisingly and contrary to our expectations, revealed that the level of DNA methylation in RPMI 1640 Folatemedium is significantly higher than DMEM/F-12 and RPMI 1640 Folate+ medium. One explanation for this observation is that the available amount of folic acid in FBS is relatively lower than the amount present in DMEM/F-12 and RPMI 1640 Folate+ medium $(6.0 \mu \mathrm{M}$ vs. $2.3 \mu \mathrm{M})$ and cells through compensatory mechanism may uptake more folic acid and have a more active one carbon metabolism cycle in compare to other groups, which needs more investigations to be elucidated.

Increasing evidence indicates that folate plays important roles in protein synthesis, cell division and growth. In addition folate is a methyl donor that plays a critical role in methylation reactions, including DNA methylation. Many in vivo studies and animal models demonstrated the relation between folate deficiency and the development of genomic DNA hypomethylation, which is an early epigenetic found in many cancers including colorectal, gastric and breast cancers.

There are few and sparse data about the folate deprivation in in vitro studies. Based on the literature, supplementation of reduced folate media with FBS can maintain the normal growth of cultured cells because FBS contains folate in an amount which is sufficient for cell survival ${ }^{17}$. Regard to this, Stempak and colleagues used dialyzed serum in order to eliminate folic acid from the serum for assessing the effect of folate deprivation on methionine cycle intermediates and DNA methylation ${ }^{15}$. Interestingly, when we reduced the serum in culture medium from $10 \%$ to $0.5 \%$, we observed that cells cultured in RPMI 1640 folic free medium (RPMI 1640 Folate-) present lower global DNA methylation level in compare to other groups. As SAM is the universal methyl donor for histone methylation and abnormal histone methylation has also been considered as a barrier to the reprogramming of bovine SCNT embryos, we also investigated the effects of folate deprivation in fibroblast donor cells on $\mathrm{H} 3 \mathrm{~K} 9 \mathrm{me} 2$ of fibroblast cells. Interestingly, we observed that the value for $\mathrm{H} 3 \mathrm{~K} 9 \mathrm{me} 2$ remained unchanged following 6 days folate deprivation in culture medium in presence of $0.5 \%$ FBS. Studies on folate deprivation impact on histone methylation are sparse. In liver samples of mouse fed with a folate-deficient diet, $\mathrm{H} 3 \mathrm{~K} 4 \mathrm{me}$ was increased but the value for $\mathrm{H} 3 \mathrm{~K} 9 \mathrm{me} 2$ remained unchanged ${ }^{18}$. A knockdown of MTHFR in HeLa cells causes a decrease in $\mathrm{H} 3 \mathrm{~K} 9 \mathrm{me} 3$ and an increase in centromeric satellite repeat expression ${ }^{19}$. Upon choline deprivation of mouse neural-progenitor cells, $\mathrm{H} 3 \mathrm{~K} 9 \mathrm{me} 2$ is modified at specific genomic locus, causing poor recruitment of $\mathrm{G} 9 \mathrm{a}^{20}$. The fact that value for $\mathrm{H} 3 \mathrm{~K} 9 \mathrm{me} 2$ is unchanged by folate deficiency in the current study, unlike the differences observed for DNA methylation, however, remained to be elucidated.

To further investigate the effect of folic insufficiency on site-specific DNA methylation of cells propagated in absence of folate, we assessed the DNA methylation of $P O U 5 F 1$ promoter as a non-imprinting gene and ICR of H19/IGF2 as an imprinting gene. While we observed a DNA hypo-methylation in POU5F1 gene, no changes were observed in ICR of $H 19 / I G F 2$. These results possibly indicate that imprinted genes are recalcitrant to epigenetic changes which is consistent with the role envisaged for imprinted genes ${ }^{21}$. Furthermore, these result suggest that promoter of genes related to pluripotency are prone to relaxation by this approach ${ }^{22,23}$.

Several studies have shown that folate deprivation reduces proliferation of various cell types ${ }^{24,25}$. Furthermore, it has been shown that cells lacking folate accumulate in the $S$ phase ${ }^{26}$ and present increased uracil mis-incorporation which eventually end in DNA damage. In accordance with previous studies, we also observed that higher percentage of cells are located in S phase in RPMI Folate- in compare to other groups which is likely related to nucleotide imbalance and slow DNA synthesis in folate deprived cells in compare to cells supplement with adequate amount of folate.

In contrary, we observed that neither type of culture medium (DMEM/F-12 or RPMI 1640) nor folate deprivation affected DNA damage in presence of 10 or $0.5 \%$ FBS. These insignificant changes may be result from short in vitro culture period and extended exposure to this condition may result in lower proliferation rate and higher DNA damage.

By assessing the effect of folic acid deprivation in RPMI 1640 medium on efficiency of SCNT, we observed a two fold increase in blastocyst rate which is in accordance with the characteristic of folate deprived cells such as reduced global and site-specific DNA methylation and increased nuclear area related to relaxation of chromatin. These observations indicate that RPMI Folate- culture medium induces a more favorable condition for better epigenetic and chromatin make up for SCNT procedure. It was also interesting to note that, while there was no difference in the various cellular characteristics of DMEM/F-12 Folate+ and RPMI 1640 Folate + medium, no difference was also observed in the efficiency of SCNT between the two medium.

Our data revealed that the epigenetic signature of POU5F1 promoter of blastocysts in RPMI Folate-group is similar to IVF group. To further verify the beneficial effect of in vitro folate deprivation, we compared the relative expression of some developmentally important genes between IVF, RPMI Folate + and RPMI Folate- groups. Gene expression analysis illustrates that the relative expression of NANOG as a gene related to developmental competency and DNMT3 required for establishing DNA methylation after DNA demethylation during preimplantation stage was higher in blastocysts from RPMI Folate - compared with RPMI 1640 Folate+ group and was similar to IVF derived blastocysts, indicating the beneficial effect of global DNA hypo-methylation induced by folate deprivation on blastocyst quality. 


\section{Conclusion}

In summary, we have established an improved SCNT method by inducing a folate deprivation in vitro. Using this method, we observed a two fold increase in blastocyst rate in RPMI 1640 Folate - group in compare to RPMI 1640 Folate + group and subsequently the derived blastocysts have similar gene expression and epigenetic signature to IVF blastocysts than those in RPMI 1640 Folate + group. The results of this study may provide a promising non-chemical approach instead of using chemical inhibitors of epigenetic modifier enzymes for improving mammalian cloning efficiency for agricultural and biomedical purposes.

\section{Materials and methods}

Media and reagents. Unless stated differently, all chemicals and media were obtained from Sigma Aldrich Chemical Co. (St. Louis, MO) and Gibco (Grand Island, NY), respectively. The Institutional Review Board and Institutional Ethical Committee of the Royan Institute approved all animal care protocols and the proposal. In addition, all methods were performed in accordance with the Institutional Review Board and Institutional Ethical Committee of the Royan Institute guidelines and regulations.

Experimental design. At passage 3-4, fibroblast cells were added in equal densities to 6-mm culture dishes containing DMEM/F12 (Gibco 32500-035, USA), RPMI 1640 Folate+ (Biowest L0498, France) and RPMI 1640 Folate- (Gibco 27016-021, USA) plus $10 \%$ or $0.5 \%$ FBS (Gibco 10270, USA). In presence of $10 \%$ FBS cells were passaged every 3 days and cultured for 6 days. For treatment of donor cells in culture medium containing $0.5 \%$ FBS, primarily they were cultured in presence of $10 \%$ FBS for 3 days and subsequently the amount of FBS was reduced to $0.5 \%$ FBS for 6 days. At the end of treatment, cells were used for analyzing of different cellular characteristics such as cell proliferation, cell cycle, DNA fragmentation, nuclear area, evaluation of DNA methylation and $\mathrm{H} 3 \mathrm{~K} 9 \mathrm{me} 2 \mathrm{using}$ immunostaining with flow cytometry, evaluation of DNA methylation of promoter of POU5F1 gene and ICR of H19/IGF2 imprinted genes using bisulphite sequencing and gene expression as described below. Finally treated cells were used for SCNT. All the experiments were carried out in triplicates.

Bovine fetal fibroblast cells (BFFs) collection. BFFs were isolated from an approximately 2-month-old female fetus. Briefly, after removal of head, limbs and viscera, remaining tissue was washed in phosphate buffered saline without calcium and magnesium $\left(\mathrm{PBS}^{-}\right.$) (Gibco 21600, USA). The tissue was finely minced using a sterile razor blade until it becomes possible to pipette. Subsequently, the minced tissue was dissociated with $0.25 \%$ trypsin/EDTA (Gibco 25300, USA) for $10 \mathrm{~min}$ at $37^{\circ} \mathrm{C}$. The cell suspension was washed, centrifuged and cultured in Dulbecco's modified Eagle medium F-12 (DMEM/F-12) containing 10\% FBS and 1\% penicillin-streptomycin (Pen/Strep) (Gibco 15140122 , USA) at $37.5^{\circ} \mathrm{C}$ and $5 \% \mathrm{CO}_{2}$ in a humidified atmosphere. After reaching confluency, BFFs were harvested and frozen. Frozen stocks were thawed and fibroblast at passage 3-4 were used for various experiments.

Cell culture of BFF donor cells. At passage 3-4, BFFs were cultured in DMEM/F-12 containing $6 \mu \mathrm{M}$ folic acid (DMEM/F-12 Folate+, control group), RPMI 1640 medium containing $2.3 \mu \mathrm{M}$ folic acid (RPMI 1640 Folate+, RMPI 1640 control group) and folic acid free RPMI 1640 medium (0 mM folic acid) (RPMI 1640 Folate-, deprived group), which were supplemented with $10 \%$ or $0.5 \%$ FBS and $1 \%$ Pen/Strep. Cells were passaged every 3 days and cultured for 6 days in presence of $10 \%$ FBS. For treatment of donor cells in culture medium containing $0.5 \%$ FBS, primarily they were cultured in presence of $10 \%$ FBS for 3 days and subsequently the amount of FBS was reduced to $0.5 \%$ FBS for 6 days.

Cell proliferation assessment. Proliferation of fibroblast cells in presence or absence of folic acid was determined using 3- (4, 5-dimethylthiazol-2-yl)-5-(3-carboxymethoxyphenyl)-2-(4-sulfophenyl)-2H-tetrazolium (MTS) assay (Promega G3582, USA). In brief, 1000 and 15000 BFF cells were cultured in presence of $10 \%$ and 0.5\% FBS in DMEM/F-12 Folate+, RPMI 1640 Folate+ and RPMI 1640 Folate-culture medium in 96-well dish. After the desired times, MTS was added to each well and incubated for $4 \mathrm{~h}$ at $37^{\circ} \mathrm{C}$. Absorbance ratio of cultured cells in RPMI 1640 Folate + and RPMI 1640 Folate - relative to DMEM/F-12 Folate + was measured at $492 \mathrm{~nm}$ by using multi-well spectrophotometer. All analyses were measured in three independent replicates and each replicate consisted of triplicate samples.

Cell cycle assessment. Cell cycle analysis was carried out by flow cytometry as described by Jafarpour et al. ${ }^{27}$. Briefly; cultured cells in different culture medium were trypsinized and resuspended in ice-cold $75 \%$ ethanol for $1 \mathrm{~h}$. The fixed cells were resuspended in staining solution containing propidium iodide $(50 \mu \mathrm{g} / \mathrm{mL})$, RNase A $(100 \mu \mathrm{g} / \mathrm{mL})$, and Triton X-100 $(0.5 \%)$ for $20 \mathrm{~min}$ in the dark at room temperature (RT). In order to remove the aggregated cells, the cell suspension was filtered through $40 \mu \mathrm{m}$ nylon mesh. Finally, a total of 20,000 stained cells were collected on a fluorescence-activated cell sorter (FACS) Caliber (Becton Dickinson, San Jose, CA) and were analyzed using Modfit software ${ }^{28}$.

DNA fragmentation analysis. To assess the effect of folate deprivation on DNA fragmentation, treated cells were stained using terminal deoxynucleotidyl transferase (TdT)-mediated dUTP-digoxigenin nick end labeling (TUNEL) with an in situ cell-death detection kit (Promega_Diagnostic Corporation, Madison, WI, USA). Briefly, according to the manufacturer's guidelines, cells were fixed with paraformaldehyde for $20 \mathrm{~min}$ at $4{ }^{\circ} \mathrm{C}$. Subsequently, cells were permeabilized with $0.2 \%$ Triton X-100 for $15 \mathrm{~min}$ at RT. After removing the permeabilization solution, cells were incubated with equilibration buffer (EB) for $5 \mathrm{~min}$ at RT. Then cells were resuspended in $50 \mu \mathrm{l}$ of $\mathrm{rTdT}$ incubation buffer $(45 \mu \mathrm{l} \mathrm{EB}+1 \mu \mathrm{l} \mathrm{rTdT}$ enzyme $+5 \mu \mathrm{l}$ nucleotide mix $)$ and incubated in a water bath for 60 minutes at $37^{\circ} \mathrm{C}$ in the dark. The reaction was stopped with $1 \mathrm{ml}$ of $20 \mathrm{mM}$ EDTA. After that, cells were 


\begin{tabular}{|c|c|c|c|c|}
\hline Gene & Forward primer $\left(5^{\prime}-3^{\prime}\right)$ & Reverse primer $\left(5^{\prime}-3^{\prime}\right)$ & $\mathbf{A T}^{*}$ & $\begin{array}{l}\text { Product } \\
\text { size }\end{array}$ \\
\hline POU5F1 & GTTAGAGGTTAAGGTTAGTGGGTG & AAAAACCCTTAAAAACTCATCCAA & $63^{\circ} \mathrm{C}$ & $235 \mathrm{bp}$ \\
\hline H19/IGF2 ICR & GGATAGGAGATTAGGTTTAGAGGG & AATCCTATAATATACAAACCCCC & $66^{\circ} \mathrm{C}$ & $225 \mathrm{bp}$ \\
\hline
\end{tabular}

Table 2. Primers used in this study for bisulfite sequencing analysis.

washed with PBS $^{-}$containing $0.1 \%$ Triton X-100 ${ }^{\circledR}$ and $5 \mathrm{mg} / \mathrm{ml}$ BSA. Finally, cells were analyzed with a FACSCalibur $^{\mathrm{TM}}$ flow cytometer (Becton Dickinson, San Jose, CA).

Nuclear area assessment. To assess the effect of presence or absence of folic acid on chromatin structure, fibroblast cells in various treatment groups were fixed and stained with Hoechst 33342. Nuclear area of 100 fibroblasts in 10 different fields was calculated using ImageJ software (National Institute of Mental Health, Bethesda, Maryland, USA).

Assessment of epigenetic marks in fibroblasts, 5-methylcytosine and H3K9me2. Flow cytometry. The effect of folic acid deprivation on global DNA methylation and H3K9me2 of fibroblast cells were assessed using immunostaining with flow cytometry through measuring the fluorescence intensity of the complexes between primary and secondary antibodies in the cells as described in our previous papers ${ }^{29}$. In brief, after trypsinization, cells were fixed with $70 \%$ ethanol for $1 \mathrm{~h}$ in $4^{\circ} \mathrm{C}$. Permeabilization was carried out using $1 \%$ Triton X-100 in PBS- for $30 \mathrm{~min}$ at RT. For detection of 5-methylcytosine DNA was denatured with $\mathrm{HCl}(4 \mathrm{~N})$ and then the effect of $\mathrm{HCl}$ was neutralized with Tris-HCL (0.1 M). Subsequently, after blocking the non-specific binding site with blocking solution for $2 \mathrm{~h}$ at RT, cells were incubated with primary antibody (5-mc, Eurogentec BI-MECY-0500, Belgium or H3K9me2, Abcam ab1220) for overnight in $4{ }^{\circ} \mathrm{C}$. After extensive washing, cells were incubated with secondary antibody (Chemicon AP124F) for $1 \mathrm{~h}$ at $37^{\circ} \mathrm{C}$. Cells were collected with the FACS-Caliber and were analyzed using CELL QUEST 3.1 software (Becton Dickinson). Three replicates were conducted for each treatment with appropriate controls to eliminate the possible effects of auto-fluorescence and nonspecific binding by the secondary antibody.

Analysis of DNA methylation using bisulfite sequencing PCR. Bisulfite sequencing was carried out based on previous studies ${ }^{30}$. In brief, genomic DNA was extracted using DNeasy ${ }^{\circledR}$ Blood and Tissue Kit (Qiagen 69504). Then DNA was treated for bisulfite sequencing according to the user manual of EpiTect ${ }^{\circledR}$ Bisulfite Kit (Qiagen, Germany).

Meth Primer online software was chosen for primer design (www.urogene.org/MethPrimer) (Table 2). The PCR master mix include $300 \mathrm{ng}$ of each forward and reverse primers, $4 \mu \mathrm{l}$ bisulfite modified DNA, $1 \times$ ammonium sulfate (AMS) buffer (Cinna Gen, Iran), $6.7 \mathrm{mM} \mathrm{MgCl}_{2}$ (Cinna Gen, Iran), $1.25 \mathrm{mM}$ dNTP (Cinna Gen, Iran), 0.6 USmar Taq (Cinna Gen) and $10 \mathrm{mM} \mathrm{2-mercaptoethanol} \mathrm{(Sigma)} \mathrm{in} \mathrm{a} 50 \mu 1$ reaction volume. PCR reaction was set based on following program: $95^{\circ} \mathrm{C}$ for $10 \mathrm{~min}, 39 \mathrm{cycles}$ at $95^{\circ} \mathrm{C}$ for $30 \mathrm{sec}, 63^{\circ} \mathrm{C}$ for $30 \mathrm{sec}$, and $72^{\circ} \mathrm{C}$ for $1 \mathrm{~min}$, followed by a final extension at $72^{\circ} \mathrm{C}$ for $10 \mathrm{~min}^{31}$. Afterward, PCR products were subcloned into a pTZ57R/T cloning vector (InsTAcloneTM PCR Cloning Kit, Fermentas) as described in the manufacturer's protocol. Ligated vectors were transferred into the $\mathrm{DH} 5 \alpha$ strain of $\mathrm{E}$. coli and at least 10 positive colonies, which were selected by PCR analysis through M13 forward and reverse primers, were extracted by Qiaprep ${ }^{\circledR}$ Spin Miniprep Kit (Qiagen) and sequenced. The sequence of primers is listed in Table 2. The sequences were analyzed with bisulfite sequencing DNA methylation analysis (BISMA) online software ${ }^{32}$.

RNA extraction and gene expression analysis in fibroblast cells. Total RNA from fibroblast cells was extracted by RNeasy Mini Kit (QIAGEN 74106) according to manufacturer's instruction. cDNA synthesis was performed with $1 \mu \mathrm{g}$ of total RNA implementing random hexamer primer and RevertAid ${ }^{\mathrm{TM}} \mathrm{H}$ First Strand cDNA Synthesis Kit (Takara RR037A). Real time PCR was performed with SYBR Green PCR Master Mix (TaKaRa) in a Thermal Cycler Rotor-Gene 6000 (Corbett, Mortlake, Australia), according to the manufacturer's protocol. Expressions of target genes were normalized to B-actin gene expression level. All measurements were carried out in triplicate, from three separate samples, and data were analyzed using the $2^{-\mathrm{ddCt}}$ method. The sequence of primers is listed in Table 3.

Preparation of bovine ovaries and oocyte collection. Ovaries were obtained from cows at local slaughterhouse (Fasaran, Isfahan), with the permission of the manager of the slaughterhouse and the agreement of veterinary organization. Ovaries were collected from slaughterhouse at 2-4 p.m. and transported to the laboratory at $15-17^{\circ} \mathrm{C}$ by $6 \mathrm{p} . \mathrm{m}$. Immediately, after receiving the ovaries, they were washed, trimmed and stored at $15^{\circ} \mathrm{C}$ until time for harvesting the oocytes based on previously set protocols ${ }^{33,34}$. The cumulus oocyte complexes (COCs) were aspirated from $2-8 \mathrm{~mm}$ follicles using an 18 gauge needle attached to a vacuum pump. Only oocytes possessing homogenous cytoplasm and at least three layers of compact cumulus cells were selected for in vitro maturation (IVM).

IVM of bovine oocyte. The procedure of IVM of oocytes was according to Hosseini et al. ${ }^{35}$. In brief, selected COCs were primarily washed in hepes-tissue culture medium 199 (H-TCM) and subsequently in TCM medium supplemented with sodium pyruvate, $10 \% \mathrm{FBS}, 10 \mu \mathrm{g} / \mathrm{ml}$ follicle stimulating hormone (FSH; Sigma F8174), $10 \mu \mathrm{g} / \mathrm{ml}$ luteinizing hormone (LH; Sigma L5269), $100 \mathrm{mM} 17 \beta$-estradiol (E2; Sigma E4389), $0.1 \mathrm{mM}$ cysteamine (Sigma M9768), $10 \mathrm{ng} / \mathrm{ml}$ epidermal growth factor (EGF; Sigma E4127) and $100 \mathrm{ng} / \mathrm{ml}$ insulin-like growth factor 


\begin{tabular}{|l|l|l|l|l|l|}
\hline Gene & Forward primer $\left(5^{\prime}-\mathbf{3}^{\prime}\right)$ & Reverse primer $\left(5^{\prime} \mathbf{3}^{\prime}\right)$ & AT* & $\begin{array}{l}\text { Product } \\
\text { size }\end{array}$ & Accession number \\
\hline$B$ - $A C T I N$ & TTCCTGGGTATGGATCCTG & GGTGATCTCCTTCTGCATCC & $58^{\circ} \mathrm{C}$ & $130 \mathrm{bp}$ & XM_015467124.1 \\
\hline$M T H F R$ & AAGATGAAGCGGAAGATG & AACCTGGAGATGAGATTG & $48^{\circ} \mathrm{C}$ & $98 \mathrm{bp}$ & NM_001011685.1 \\
\hline$B H M T$ & CAGACCTTCACCTTCTATGC & CTCCTTCATCAGCCACTTG & $56^{\circ} \mathrm{C}$ & $130 \mathrm{bp}$ & NM_001011679.1 \\
\hline POU5F1 & GGAAAGGTGTTCAGCCA & ATTCTCGTTGTTGTCAGC & $62^{\circ} \mathrm{C}$ & $123 \mathrm{bp}$ & NM_174580.3 \\
\hline NANOG & TTGTGACGGCTATTGTATG & ACCTCTTACTGGACTCATT & $53^{\circ} \mathrm{C}$ & $159 \mathrm{bp}$ & NM_001025344.1 \\
\hline DNMT3a & TGGTCCTGGGCGTTAG & CCTGCTTTATGGAGTTCG & $57^{\circ} \mathrm{C}$ & $252 \mathrm{bp}$ & NM_001206502.1 \\
\hline$H 19$ & TCAGCCCCGAGACCAC & ACGCTCAGAGACCAGG & $53^{\circ} \mathrm{C}$ & $327 \mathrm{bp}$ & XM_001256398.4 \\
\hline IGF2 & CCTGCTGGAGACTTACTG & CTTGGCGAGCGTGCGA & $62^{\circ} \mathrm{C}$ & $199 \mathrm{bp}$ & XM_015461332.1 \\
\hline
\end{tabular}

Table 3. List of primers used in this study for real time PCR.

1 (IGF1; R\&D 291-G1) (maturation medium). COCs were then transferred and cultured in groups of 10 into $50 \mu \mathrm{l}$ droplets of maturation medium and incubated for $22-24 \mathrm{~h}$ at $38.5^{\circ} \mathrm{C}$ in a humidified $5 \% \mathrm{CO}_{2}$ atmosphere under mineral oil.

In vitro fertilization (IVF) of bovine. In this study, IVF was carried out as a control group. The IVF procedure was as described previously (Hosseini et al., 2016). Motile sperms were collected using swim down method with PureSperm ${ }^{\circledR}$ gradient. $1 \times 10^{6} / \mathrm{ml}$ sperms were co-incubated with 10 matured COCs in $50 \mu l$ fertilization medium for $18 \mathrm{~h}$ at $38.5^{\circ} \mathrm{C}, 5 \% \mathrm{CO}_{2}$ and humidified atmosphere. After $18 \mathrm{~h}$, presumptive zygotes were denuded of cumulus cells and cultured in BO-IVC medium (IVF Bioscience) at $38.5^{\circ} \mathrm{C}, 5 \% \mathrm{CO}_{2}, 5 \% \mathrm{O}_{2}$ and humidified air for 7-8 days under mineral oil. Derived blastocysts were used for Bisulfite sequencing PCR and gene expression analysis.

SCNT of bovine. After denudation of matured oocytes with hyaluronidase and by vortexing, denuded oocytes were exposed to $5 \mathrm{mg} / \mathrm{ml}$ pronase for few seconds for removal of zona pellucida. The method of oocyte enucleation was done using manual oocyte enucleation using a fine pulled Pasteur pipette ${ }^{36}$. Briefly, zona free oocytes were incubated in TCM supplemented with $4 \mu \mathrm{g} / \mathrm{ml} \mathrm{demecolcine} \mathrm{for} 1 \mathrm{~h}$ in $38.5^{\circ} \mathrm{C}$. Then, cytoplasmic protrusion containing MII spindle, was removed by hand-held manual oocyte enucleation pipette. The procedures of nuclear replacement, electrofusion, oocyte activation were the same as described in preceding papers ${ }^{36}$. Subsequently, activated reconstructed oocytes were cultured inside well containing BO-IVC (IVF Bioscience) at $38.5^{\circ} \mathrm{C}, 5 \% \mathrm{CO}_{2}, 5 \% \mathrm{O}_{2}$ and humidified air for 7-8 days under mineral oil. To preclude aggregation of zona-free embryos, $20 \mu \mathrm{l}$ droplets of BO-IVC were prepared under mineral oil in $3 \mathrm{~cm}$ Grainer ${ }^{\circledR}$ dishes. Six small wells were made by gently pressing a sterile steel rod with a round tip to the bottom of the culture dish and activated reconstructed oocytes were put in separate wells.

Bisulfite sequencing PCR in IVF and SCNT derived blastocysts. Genomic DNA of blastocysts was extracted by salting-out method. All the detailed procedures of bisulfite sequencing PCR were exactly the same as mentioned for fibroblast cells in previous section.

RNA extraction and Gene expression analysis. Total RNA from blastocysts was extracted by RNeasy Micro Kit (QIAGEN, cat.No. 74004) respectively according to manufacturer's instruction. All the detailed procedures of RNA extraction and Gene expression analysis were exactly the same as mentioned for fibroblast cells in previous section.

Statistical analysis. All experiments were repeated at least three times. Data is presented as mean \pm S.E.M. Statistical significance was set at $P<0.05$. One-way analyses of variance (ANOVA) was applied to study the effects of folate among various treatment groups $(\alpha=0.05)$ followed by Tukey post-hoc test. The mRNA expression of MTHFR and BHMT genes in bovine fibroblast and kidney cells, and DNA methylation and mRNA expression of assessed genes in BFF-RPMI Folate + and Folate - were analysed by independent samples t-test. GraphPad Prism (v.6.0.1) and IBM SPSS program (v.23, NY, USA) were used for statistical analysis.

Received: 21 June 2019; Accepted: 2 March 2020;

Published online: 19 March 2020

\section{References}

1. Akagi, S., Geshi, M. \& Nagai, T. Recent progress in bovine somatic cell nuclear transfer. Anim Sci J. 3, 191-199 (2013)

2. Wilmut, I., Schnieke, A. E., McWhir, J., Kind, A. J. \& Campbell, K. H. Viable offspring derived from fetal and adult mammalian cells. Nature 385, 810 (1997).

3. Rodriguez-Osorio, N., Urrego, R., Cibelli, J., Eilertsen, K. \& Memili, E. Reprogramming mammalian somatic cells. Theriogenology 78, 1869-1886 (2012).

4. Dean, W. et al. Conservation of methylation reprogramming in mammalian development: aberrant reprogramming in cloned embryos. Proceedings of the National Academy of Sciences 98, 13734-13738 (2001).

5. Santos, F. et al. Epigenetic marking correlates with developmental potential in cloned bovine preimplantation embryos. Current Biology 13, 1116-1121 (2003).

6. Yoshida, M., Kijima, M., Akita, M. \& Beppu, T. Potent and specific inhibition of mammalian histone deacetylase both in vivo and in vitro by trichostatin A. Journal of Biological Chemistry 265, 17174-17179 (1990). 
7. El Kharroubi, A., Piras, G. \& Stewart, C. L. DNA demethylation reactivates a subset of imprinted genes in uniparental mouse embryonic fibroblasts. Journal of Biological Chemistry 276, 8674-8680 (2001).

8. Enright, B. P., Kubota, C., Yang, X. \& Tian, X. C. Epigenetic characteristics and development of embryos cloned from donor cells treated by trichostatin A or 5-aza-2'-deoxycytidine. Biol Reprod 69, 896-901 (2003).

9. Blelloch, R. et al. Reprogramming efficiency following somatic cell nuclear transfer is influenced by the differentiation and methylation state of the donor nucleus. Stem Cells 24, 2007-2013 (2006).

10. Lee, M. B. et al. Betaine homocysteine methyltransferase is active in the mouse blastocyst and promotes inner cell mass development. Journal of Biological Chemistry 287, 33094-33103 (2012).

11. Delgado-Reyes, C. V., Wallig, M. A. \& Garrow, T. A. Immunohistochemical detection of betaine-homocysteine S-methyltransferase in human, pig, and rat liver and kidney. Archives of Biochemistry and Biophysics 393, 184-186 (2001).

12. Selhub, J. Homocysteine metabolism. Annual review of nutrition 19, 217-246 (1999).

13. Locasale, J. W. Serine, glycine and one-carbon units: cancer metabolism in full circle. Nature reviews Cancer 13, 572 (2013).

14. Bishop, K. S. \& Ferguson, L. R. The interaction between epigenetics, nutrition and the development of cancer. Nutrients 7, 922-947 (2015).

15. Stempak, J. M., Sohn, K. J., Chiang, E. P., Shane, B. \& Kim, Y. I. Cell and stage of transformation-specific effects of folate deficiency on methionine cycle intermediates and DNA methylation in an in vitro model. Carcinogenesis 26, 981-990 (2005).

16. Wang, J., Dudman, N. P., Lynch, J. \& Wilcken, D. E. Betaine: homocysteine methyltransferase-a new assay for the liver enzyme and its absence from human skin fibroblasts and peripheral blood lymphocytes. Clinica Chimica Acta. 204, 239-249 (1991).

17. Nadeau, J. L. et al. Quantum dot systems for specific biosensing applications. In Colloidal Quantum Dots for Biomedical Applications II. International Society for Optics and Photonics 6448, 64480M (2007).

18. García-Compeán, D. et al. Current concepts in diabetes mellitus and chronic liver disease: clinical outcomes, hepatitis $C$ virus association, and therapy. Digestive diseases and sciences 61, 371-380 (2016).

19. Zhu, B. et al. MTHFR promotes heterochromatin maintenance. Biochemical and biophysical research communications 447, 702-706 (2014).

20. Mehedint, M. G., Craciunescu, C. N. \& Zeisel, S. H. Maternal dietary choline deficiency alters angiogenesis in fetal mouse hippocampus. Proceedings of the National Academy of Sciences 107, 12834-12839 (2010).

21. Cerrato, F. et al. Paternal imprints can be established on the maternal Igf2-H19 locus without altering replication timing of DNA. Human molecular genetics 12, 3123-3132 (2003)

22. Smallwood, S. A. et al. Dynamic CpG island methylation landscape in oocytes and preimplantation embryos. Nature genetics 43, 811 (2011).

23. Obata, Y. \& Kono, T. Maternal primary imprinting is established at a specific time for each gene throughout oocyte growth. Journal of Biological Chemistry 277, 5285-5289 (2002).

24. Kruman, I. I., Mouton, P. R., Emokpae, R. Jr., Cutler, R. G. \& Mattson, M. P. Folate deficiency inhibits proliferation of adult hippocampal progenitors. Neuroreport 16, 1055-1059 (2005)

25. Craciunescu, C. N. et al. Folic acid deficiency during late gestation decreases progenitor cell proliferation and increases apoptosis in fetal mouse brain. The Journal of nutrition 134, 162-166 (2004).

26. Courtemanche, C., Elson-Schwab, I., Mashiyama, S. T., Kerry, N. \& Ames, B. N. Folate deficiency inhibits the proliferation of primary human CD8+ T lymphocytes in vitro. The Journal of Immunology 173, 3186-3192 (2004).

27. Jafarpour, F. et al. Somatic cell-induced hyperacetylation, but not hypomethylation, positively and reversibly affects the efficiency of in vitro cloned blastocyst production in cattle. Cellular Reprogramming (Formerly "Cloning and Stem Cells") 13, 483-493 (2011).

28. Boquest, A. C., Day, B. N. \& Prather, R. S. Flow cytometric cell cycle analysis of cultured porcine fetal fibroblast cells. Biology of reproduction 60, 1013-1019 (1999).

29. Pezhman, M. et al. Cathepsin B inhibitor improves developmental competency and cryo-tolerance of in vitro ovine embryos. BMC developmental biology 17, 10 (2017).

30. Derakhshan-Horeh, M. et al. Vitrification at Day3 stage appears not to affect the methylation status of H19/IGF2 differentially methylated region of in vitro produced human blastocysts. Cryobiology 73, 168-174 (2016).

31. Herman, J. G., Graff, J. R., Myöhänen, S., Nelkin, B. D. \& Baylin, S. B. Methylation-specific PCR: a novel PCR assay for methylation status of CpG islands. Proceedings of the national academy of sciences 93, 9821-9826 (1996).

32. Rohde, C., Zhang, Y., Reinhardt, R. \& Jeltsch, A. BISMA-Fast and accurate bisulfite sequencing data analysis of individual clones from unique and repetitive sequences. BMC bioinformatics 11, 230 (2010).

33. Pezhman, M. et al. Cathepsin B inhibitor improves developmental competency and cryo-tolerance of in vitro ovine embryos. BMC developmental biology 17, 10 (2017).

34. Sefid, F. et al. Vitamin K2 improves developmental competency and cryo-tolerance of in vitro derived ovine blastocyst. Cryobiology 77, 34-40 (2017)

35. Hosseini, S. M. et al. Epigenetic modification with trichostatin A does not correct specific errors of somatic cell nuclear transfer at the transcriptomic level; highlighting the non-random nature of oocyte-mediated reprogramming errors. BMC genomics 17, 16 (2016).

36. Hosseini, S. M. et al. Cloned sheep blastocysts derived from oocytes enucleated manually using a pulled pasteur pipette. Cellular Reprogramming (Formerly “Cloning and Stem Cells") 15, 15-23 (2013).

\section{Acknowledgements}

The authors would like to express their gratitude to Royan Institute for their full support. This study was funded by a grant from Royan Institute, Iran, Islamic Republic of.

\section{Author contributions}

F.J. and M.H.N.E. conceived and designed the study. F.J. and M.H.N.E. Conducted the research. M.J., R.M., F.GH.Z. and K.H.K. Performed the experiments. M.J. and F.J. analyzed the data. F.J. and M.H.N.E. wrote the manuscript.

\section{Competing interests}

The authors declare no competing interests.

\section{Additional information}

Correspondence and requests for materials should be addressed to F.J. or M.H.N.-E.

Reprints and permissions information is available at www.nature.com/reprints. 
Publisher's note Springer Nature remains neutral with regard to jurisdictional claims in published maps and institutional affiliations.

(c) (i) Open Access This article is licensed under a Creative Commons Attribution 4.0 International License, which permits use, sharing, adaptation, distribution and reproduction in any medium or format, as long as you give appropriate credit to the original author(s) and the source, provide a link to the Creative Commons license, and indicate if changes were made. The images or other third party material in this article are included in the article's Creative Commons license, unless indicated otherwise in a credit line to the material. If material is not included in the article's Creative Commons license and your intended use is not permitted by statutory regulation or exceeds the permitted use, you will need to obtain permission directly from the copyright holder. To view a copy of this license, visit http://creativecommons.org/licenses/by/4.0/.

(c) The Author(s) 2020 